\title{
HVMANITAS
}

\section{La formazione del metodo aristotelico della dimostrazione}

Autor(es): Tarantino, Piero

Publicado por: Faculdade de Letras da Universidade de Coimbra, Instituto de Estudos

URL

persistente: URI:http://hdl.handle.net/10316.2/22919

DOI: $\quad$ DOI:http://dx.doi.org/10.14195/2183-1718_63_8

Accessed : $\quad$ 26-Apr-2023 00:42:07

A navegação consulta e descarregamento dos títulos inseridos nas Bibliotecas Digitais UC Digitalis, UC Pombalina e UC Impactum, pressupõem a aceitação plena e sem reservas dos Termos e Condições de Uso destas Bibliotecas Digitais, disponíveis em https://digitalis.uc.pt/pt-pt/termos.

Conforme exposto nos referidos Termos e Condições de Uso, o descarregamento de títulos de acesso restrito requer uma licença válida de autorização devendo o utilizador aceder ao(s) documento(s) a partir de um endereço de IP da instituição detentora da supramencionada licença.

Ao utilizador é apenas permitido o descarregamento para uso pessoal, pelo que o emprego do(s) título(s) descarregado(s) para outro fim, designadamente comercial, carece de autorização do respetivo autor ou editor da obra.

Na medida em que todas as obras da UC Digitalis se encontram protegidas pelo Código do Direito de Autor e Direitos Conexos e demais legislação aplicável, toda a cópia, parcial ou total, deste documento, nos casos em que é legalmente admitida, deverá conter ou fazer-se acompanhar por este aviso. 
humanitas

Vol. LXIII

2011 


\title{
LA FORMAZIONE DEL METODO ARISTOTELICO DELLA DIMOSTRAZIONE
}

\author{
Piero Tarantino
}

\section{Sintesi}

Negli Analitici secondi di Aristotele è possibile identificare un metodo apodittico generale ed un più specifico modello dimostrativo, denominato "deduzione scientifica". Nel mio articolo riconduco questa differenza metodologica all'evoluzione del pensiero aristotelico da una preliminare teoria dell'assiomatizzazione a un procedimento sillogistico applicato alla scienza.

Parole chiave - Aristotele, Analitici secondi, dimostrazione, assiomatizzazione, deduzione scientifica.

\section{Abstract}

In Aristotle's Posterior Analytics we may find a general apodictic method and a more specific demonstrative structure, named "scientific deduction". In my paper I suggest that this methodological difference reflects the evolution of Aristotle's thought from a preliminary theory of axiomatization to a syllogistic model applied to science.

Keywords: Aristotle, Posterior Analytics, demonstration, axiomatization, scientific deduction.

1. Ogni tentativo di ricostruzione della dottrina aristotelica della dimostrazione sembra imbattersi in significativi problemi di natura filosofica e filologica, dal momento che negli Analitici secondi la formulazione del metodo apodittico, di cui si dovrebbe avvalere la scienza, si rivela non sempre coerente e lineare. L'esposizione della teoria della scienza risente 
indubbiamente della struttura compositiva degli Analitici secondi. Nell'opera sono raccolte le annotazioni aristoteliche sul tema della dimostrazione, disposte secondo un ordine che verosimilmente prescinde dalla datazione cui esse risalgono. Il criterio di una generica unità tematica sembra prevalere su una fedele successione cronologica delle sezioni del testo. Nella prima parte del trattato sono così giustapposte numerose considerazioni sulla dimostrazione, le quali, dopo una prima elaborazione, furono oggetto di parziali revisioni e di frequenti aggiunte, apportate da Aristotele in un ampio arco di tempo ${ }^{1}$.

Gli Analitici secondi, anziché fornire la presentazione ordinata di un singolo modello apodittico, raccolgono le numerose e talvolta contraddittorie tracce della riflessione aristotelica sulla scienza. La singolare costruzione della stesura degli Analitici secondi si rivela tuttavia un dato prezioso al fine di fare luce sulla formazione della teoria della dimostrazione. Il chiarimento della sovrapposizione nell'opera delle differenti fasi del pensiero scientifico aristotelico potrebbe permettere di ricostruire il percorso seguito dall'autore nella definizione di uno schema generale per l'argomentazione scientifica.

L'individuazione dei principali nuclei concettuali della dottrina apodittica, a partire dai quali si potrebbe tentare di individuare l'evoluzione del pensiero aristotelico, comporta in primo luogo una lettura selettiva dei passi degli Analitici secondi strettamente dedicati all'esposizione degli elementi apodittici. Due in particolare appaiono i momenti più significati nella riflessione aristotelica sulla dimostrazione, ovvero l'elaborazione del modello della deduzione scientifica in APo. A 2, 71b9-72a7 e la delineazione di un preliminare metodo apodittico in APo. A 7, 75a39-b2 e A 10, 76b11-22².

2. Negli Analitici secondi Aristotele formula la definizione di conoscenza scientifica, identificando nel più generale ambito gnoseologico un tipo di sapere che si costituisce mediante dimostrazione. La locuzione epistasthai haplos è scelta da Aristotele per esprimere l'avere conoscenza

${ }^{1}$ Cfr. Barnes 1994: xv.

${ }^{2}$ Le definizioni degli elementi dimostrativi, contenute nei primi capitoli degli Analitici secondi, si possono verosimilmente collegare ad almeno due schemi apodittici differenti, di cui qui si tenta di mostrare la compresenza nel trattato aristotelico. Nel presente contributo la ricostruzione e l'esame dei due modelli scientifici precedono il loro confronto e la formulazione di un'ipotesi interpretativa tesa a chiarire la costruzione della dottrina dimostrativa degli Analitici secondi. 
della ragione di qualcosa e l'avere conoscenza dell'impossibilità che questo qualcosa accada in altro modo. Essa pertanto circoscrive una forma di sapere che si caratterizza come esplicativa e necessaria ${ }^{3}$.

La comprensione della ragione (aitia) per cui qualcosa è in un determinato modo connota in modo peculiare l'episteme. Conoscere scientificamente l'eclissi di luna per esempio significa sapere che essa è determinata dall'interposizione della terra tra il sole e la luna. La ragione per cui si verifica l'eclissi lunare costituisce la spiegazione dell'evento ${ }^{4}$. L'espressione "avere conoscenza scientifica in senso proprio" (epistasthai haplos) corrisponde ad una forma di sapere più complessa rispetto alla usuale forma di conoscenza, limitata alla semplice constatazione di qualcosa.

La nozione di conoscenza scientifica (episteme) è costitutivamente collegata alla nozione di dimostrazione (apodeixis). La conoscenza scientifica si acquisisce per mezzo della dimostrazione, la quale è una particolare forma di deduzione (sulloghismos), denominata appunto "scientifica" (epistemonikos $)^{5}$. In generale la deduzione è una struttura formale in cui, posti alcuni elementi, segue necessariamente qualcosa di diverso ${ }^{6}$. Essa ha un grado di universalità maggiore rispetto alla dimostrazione: se è vero che tutte le dimostrazioni sono deduzioni, una deduzione per essere scientifica deve rispondere a requisiti specifici ${ }^{7}$.

Aristotele adotta il sillogismo come apparato logico della scienza, adeguandolo ad esprimerne i contenuti. La dottrina della dimostrazione sembra porsi come un capitolo della teoria generale dell'inferenza, dal momento che, secondo quanto esposto in APo. A 2, essa dovrebbe essere completamente sillogistica. La scienza aristotelica ha un carattere proposizionale, poiché mira ad esaminare le connessioni tra soggetto e predicato che strutturano le premesse e la conclusione di una dimostrazione.

Sul piano logico la specifica funzione esplicativa, che contraddistingue la conoscenza scientifica, è assolta dai principi della dimostrazione, i quali corrispondono alla trascrizione linguistica delle ragioni per cui si verifica

${ }^{3}$ Aristot. APo. A 2, 71b9-12. Sulla nozione di "conoscenza scientifica" negli Analitici secondi cfr. Burnyeat 1981.

${ }^{4}$ Aristot. APo. B 2, 90a14-8; Cael. B 14, 297b28-30; Metaph. H 4, 1044b13-5.

${ }^{5}$ Aristot. APo. A 2, 71b16-9.

${ }^{6}$ Aristotele fornisce la definizione tecnica di sullogismos in $A P r$. A 1, 24b 18-22.

${ }^{7}$ La maggiore generalità del sillogismo rispetto alla dimostrazione è espressa in Aristot. APr. A 4, 25b26-31. 
quanto descritto nella conclusione. Aristotele pertanto si propone di salvaguardare i presupposti su cui si basa il sapere scientifico, in quanto dalla natura dei fondamenti della deduzione dipende la scientificità delle conclusioni.

L'approfondimento della prima condizione della conoscenza scientifica, vale a dire la capacità di essere esplicativa, coincide con la caratterizzazione dei principi apodittici. I contenuti della scienza dimostrativa (apodeiktike episteme) sono acquisiti a partire da proposizioni che devono essere da una parte vere, prime e immediate, dall'altra più note, anteriori ed esplicative della conclusione ${ }^{8}$. Il primo gruppo comprende le caratteristiche appartenenti alle premesse assunte in senso assoluto, il secondo include le caratteristiche delle premesse considerate in relazione alla conclusione.

I principi (arkhai) devono essere appropriati (oikeiai) a quanto provato, vale a dire adeguati alla dimostrazione, poiché capaci di esprimere in modo conveniente la ragione dell'accadere di un evento9. L'essere vere, prime e immediate sono caratteristiche necessarie ma non sufficienti delle premesse. I principi della dimostrazione devono essere pertinenti e strettamente connessi alla conclusione. Le argomentazioni non fondate su principi appropriati sono vuote: premesse troppo generali rispetto alla conclusione non sono adatte a fornire la ragione di un fatto specifico. Non si può spiegare per esempio la sterilità del mulo a partire da un principio riguardante tutti gli animali, quale la fecondità della prole nata da genitori della stessa specie $^{10}$. Le premesse scientifiche dunque si configurano sia come principi in senso assoluto sia come ragioni della conclusione. Una deduzione che dispone di premesse prive dei suddetti requisiti non può produrre scienza ${ }^{11}$.

Aristotele esamina analiticamente le caratteristiche dei principi della scienza, dopo averle enunciate. Egli considera innanzitutto la verità delle premesse della dimostrazione: le premesse devono essere vere, cioè esprismenti uno stato di fatto, in quanto non si può avere conoscenza scientifica di ciò che non si verifica, per esempio che la diagonale è commensurabile al lato del quadrato ${ }^{12}$. I principi della dimostrazione devono essere anche primi ed immediati, ovvero indimostrabili (anapodeikta) ${ }^{13}$, in quanto non

\footnotetext{
${ }^{8}$ Aristot. APo. A 2, 71b19-22.

${ }^{9}$ Aristot. APo. A 2, 71b22-3.

${ }^{10}$ Aristot. GA. B 8, 747b30-748a14.

${ }^{11}$ Aristot. APo. A 2, 71b23-5.

${ }^{12}$ Aristot. APo. A 2, 71b25-6.

${ }^{13}$ Aristot. APo. A 2, $71 \mathrm{~b} 27$.
} 
richiedono di essere provati. Se avere conoscenza scientifica di proposizioni che sono dimostrabili significa possederne la dimostrazione, quest'ultima deve essere possibile a partire da principi primi e immediati, vale a dire non risultanti da una ulteriore deduzione ${ }^{14}$.

Le premesse della dimostrazione devono essere esplicative della conclusione, poiché si ha conoscenza scientifica di qualcosa nel momento in cui se ne conosce la ragione. Esse devono pertanto essere anteriori (protera), più note (gnorimotera) e conosciute prima (proghinoskomena) della conclusione. I principi hanno infatti una priorità gnoseologica rispetto alla conclusione sia per quanto riguarda la comprensione del loro significato sia per quanto riguarda il sapere che esistono ${ }^{15}$.

La seconda condizione della conoscenza scientifica, ovvero la necessità, è presa in considerazione nel capitolo quarto degli Analitici secondi, dedicato allo studio delle relazioni che intercorrono tra gli elementi di una proposizione autenticamente scientifica. Compito dell'uomo di scienza è lo studio di eventi universali e necessari, i quali sono espressi sul piano linguistico da particolari tipi di relazione tra un soggetto e un predicato. Nello studio dei nessi proposizionali la predicazione "per sé" o "in virtù di sé" (kath' hauto) ha una rilevanza centrale per quanto riguarda l'argomentazione scientifica. Più precisamente, sebbene siano ravvisabili quattro modi in cui $A$ può appartenere per sé a $B$, Aristotele ammette esplicitamente che solo i primi due concorrono in modo diretto alla caratterizzazione delle proposizioni dimostrative ${ }^{16}$.

In un primo senso si dicono predicati "per sé" le determinazioni che sono assunte nella definizione del soggetto. "Animale" è predicato per sé di "uomo", in quanto nella definizione di "uomo" è inclusa la proprietà "animale". I predicati "per sé" sono costitutivi del discorso definitorio del soggetto, poiché, da un punto di vista sostanziale, sono le determinazioni immanenti all'essenza, proprio come nel caso del punto rispetto alla linea e della linea rispetto al triangolo ${ }^{17}$.

${ }^{14}$ Aristot. APo. A 2, 71b26-9.

${ }^{15}$ Aristot. APo. A 2, 71b29-33. Per un'analisi dettagliata dei passi degli Analitici secondi in cui viene presentata la deduzione scientifica si rimanda ai seguenti studi: Barnes 1994: 89-103; Detel 1993: 37-85; McKirahan 1992: 23-35; Mignucci 1975: 16-43; Ross 1949: 507-12. Utili sono anche le osservazioni contenute in Mendell 1988: 161-225.

${ }^{16}$ Aristot. APo. A 4, 73b16-18; A 6, 74b5-12; A 22, 84a12-7.

${ }^{17}$ Aristot. APo. A 4, 73a34-7. 
In un secondo senso si dicono "per sé" le determinazioni che assumono nella propria definizione la nozione o il nome della cosa di cui sono proprietà. Esse di conseguenza non si possono spiegare indipendentemente dall'oggetto cui rimandano. "Pari" e "dispari" sono predicati per sé del "numero", non perché "pari" e "dispari" sono inclusi nella definizione di "numero", ma perché il "numero" è ciò a cui "pari” e "dispari" rinviano, essendo esso presente nel discorso definitorio di entrambi. Nella proposizione "l'uomo è bianco" al contrario "bianco" non è predicato per sé di "uomo", dal momento che il soggetto o la sostanza cui rimanda non è "uomo"18.

Le definizioni fornite oscillano tra il piano ontologico e il piano linguistico, mettendo in luce l'isomorfismo tipico dell'impostazione aristotelica: il kath' hauto indica sia il modo di essere di una proprietà in relazione all'essenza sia il modo di essere di un predicato in rapporto al soggetto ${ }^{19}$.

3. Negli Analitici secondi sono precisati alcuni elementi che, pur essendo strettamente attinenti alla dimostrazione, non sembrano esplicitamente collegati al modello della deduzione scientifica. Essi possono essere sinteticamente presentati, seguendo l'ordine di apparizione nel testo.

- Distinzione dei tipi di preconoscenza necessari in ogni apprendimento e in ogni acquisizione intellettuale: preconoscenza del fatto che una cosa è; preconoscenza del significato della parola che designa la cosa; preconoscenza sia del fatto che una cosa è sia del significato della parola che la designa (APO. A 1, 71a11-7).

- Distinzione dei principi immediati della deduzione in thesis e axioma: la thesis è una proposizione che non è possibile dimostrare e che non è necessario che possieda chi apprende qualcosa; lo axioma è una proposizione che è necessario che possieda chi apprende qualunque cosa. Le theseis si dividono a loro volta in hupotheseis e horismoi: la hupothesis è una proposizione con cui si ammette l'esistenza o la non esistenza di una cosa; lo horismos è una proposizione con cui è posto il significato di una espressione,

${ }^{18}$ Il terzo e il quarto modo in cui qualcosa si definisce "per sé" sono esaminati da Aristotele in APo. A 4, 73b5-16.

${ }^{19}$ Per un approfondimento della trattazione aristotelica sulle connessioni predicative si rinvia ai seguenti studi: Barnes 1994: 110-22; Detel 1993: 99-135; McKirahan 1992: 80-102; Mignucci 1975: 55-85; Ross 1949: 517-23. 
senza assumere l'esistenza o la non esistenza dell'oggetto denotato dell'espressione (APo. A 2, 72a14-24) ${ }^{20}$.

- Definizione di principio come ciò di cui non è possibile dimostrare l'esistenza. Distinzione tra oggetti primi (ta prota), di cui si assume sia l'esistenza sia la definizione, e oggetti che dipendono dai primi (ta ek touton), di cui si assume la definizione e si dimostra l'esistenza. I principi si dividono in propri (idia) di ciascuna scienza e in comuni (koina) a più scienze sulla base di un'analogia (kat' analoghian). I principi propri sono sia le assunzioni e le definizioni degli oggetti, su cui verte la dimostrazione, sia le definizioni delle proprietà $^{1}$ (APo. A 10, 76a31-b2; A 11, 77a27-8; A 32, 88b27-9).

- Enunciazione dei tre elementi costitutivi della dimostrazione: il genere sottoposto (to ghenos to hupokeimenon), intorno a cui verte la prova; gli assiomi (axiomata), proposizioni da cui si sviluppa la deduzione; le affezioni per sé (pathe), attributi di cui si dimostra l'appartenenza al genere (APo. A 7, 75a39-b2; A 10, 76b11-22).

Le sezioni degli Analitici secondi, precedentemente elencate, dedicate alla caratterizzazione delle premesse apodittiche, appaiono in larga misura come parti autonome e senza una chiara continuità rispetto al modello della deduzione scientifica illustrato in APo. A 2, 71b9-72a7. Dal momento che gli elementi definiti non sembrano contribuire ad arricchire in modo pertinente il quadro della deduzione scientifica, essi potrebbero essere stati formulati in riferimento ad un differente schema di inferenza, di cui Aristotele avrebbe fornito una prima e complessiva esposizione in APo. A 7, 75a39-b2. Il modello possiede specifici caratteri distintivi, ravvisabili fondamentalmente in tre elementi:

(a) ciò che si dimostra (to apodeiknumenon), vale a dire il termine corrispondente al predicato contenuto nella conclusione (sumpe-

${ }^{20}$ Nell'indicare gli elementi della dimostrazione si è preferito usare nella maggior parte dei casi le rispettive denominazioni greche, le quali coprono un ambito semantico non sempre adeguatamente colto dai vocaboli tradizionalmente adottati come corrispondenti nella lingua italiana.

${ }^{21}$ Per un chiarimento della complessa questione relativa alla possibile introduzione, da parte di Aristotele, di una distinzione tra termini primi e termini derivati da una parte e proposizioni prime e proposizioni derivate dall'altra si rimanda a Barnes 1994: 137-38 e a Mignucci 2007: 180-81, alle cui proposte interpretative mi sono attenuto. 
rasma $)^{22}$, la quale esprime l'appartenenza di un accidente per sé ad un genere (ghenos);

(b) gli assiomi (axiomata), proposizioni a partire da cui (ex hon) si sviluppa la dimostrazione;

(c) il genere sottoposto (to ghenos to hupokeimenon), cioè il soggetto contenuto nella conclusione, le cui affezioni (pathe) o accidenti per sé (kath' hauta sumbebekota), sono resi evidenti dalla dimostrazione ${ }^{23}$.

Lo schema è riproposto in APo. A 10, 76b11-6, sebbene cambi l'ordine di presentazione dei componenti:

(a) gli oggetti posti come esistenti, i quali costituiscono il genere, di cui la scienza studia gli attributi per sé;

(b) gli assiomi chiamati comuni, vale a dire principi primi da cui si dimostra;

(c) le affezioni, rispetto alle quali la scienza assume che cosa significhino ${ }^{24}$.

La presentazione degli elementi dimostrativi, contenuta in APo. A 10, è più particolareggiata rispetto allo schema tracciato in $A P o$. A 7. Aristotele caratterizza il genere in termini di esistenza, richiede l'assunzione della definizione in riferimento alle affezioni e qualifica gli assiomi non più semplicemente come principi ex hon, ma anche come comuni (koina) e primi (prota). In una dimostrazione inoltre si può tralasciare la menzione di alcuni dei tre elementi elencati, qualora appaiano evidenti ${ }^{25}$. La struttura tripartita della prova non è tuttavia messa in discussione, dal momento che essa si compone delle seguenti parti: (a) ciò su cui verte la dimostrazione (peri ho te deiknusi), cioè il genere; (b) le cose che si dimostrano (ha deiknusi), cioè le affezioni per sé; (c) le proposizioni da cui parte la dimostrazione (ex hon), cioè gli assiomi ${ }^{26}$.

${ }^{22}$ Ciò che si deve dimostrare è il predicato della conclusione e non la conclusione nella sua interezza, come lascerebbe intendere il termine sumperasma adoperato da Aristotele. Il riferimento al predicato della conclusione è confermato dall'inciso in APo. A 7, 75a40-1 e dalla più precisa tripartizione degli elementi della dimostrazione presentata in APo. A 10, 76b11-6.

${ }^{23}$ Aristot. APO. A 7, 75a39-b2.

${ }^{24}$ Aristot. APo. A 10, 76b11-6.

${ }^{25}$ Aristot. APo. A 10, 76b16-21.

${ }^{26}$ Aristot. APo. A 10, 76b21-2. Aristotele ribadisce spesso che in una dimostrazione ci sono per natura (a) cose su cui, (b) cose di cui e (c) cose da cui è la prova (APo. A 11, 77a27-8; A 32, 88b27-9; Metaph. B 2, 997a8-9; B 2, 997a19-21; 
Lo scopo della prova, elaborata in APo. A 7 e 10, è la verifica dell'esistenza di una proprietà, in quanto appartenente ad un oggetto che rientra in un determinato genere disciplinare ${ }^{27}$. Aristotele non fornisce una esemplificazione del modello formulato e pertanto ogni tentativo di ricostruzione resta altamente speculativo. Con le dovute cautele del caso, si può però supporre una plausibile applicazione del procedimento. Secondo le indicazioni fornite da Aristotele, nella fase preliminare si dovrebbe procedere da un lato ad assumere l'esistenza e la definizione dell'oggetto e dall'altro la definizione della proprietà. Nella fase operativa gli assiomi, proposizioni a partire da cui è svolta l'inferenza, avrebbero il compito di rendere evidente che la proprietà compete all'oggetto in qualità di sua determinazione essenziale ${ }^{28}$. La proprietà risulterebbe esistente, poiché inerisce ad un oggetto di cui si è precedentemente ipotizzata l'esistenza. La suddetta conclusione si conseguirebbe mostrando che la proprietà da verificare come esistente è legata all'oggetto della prova da una relazione di natura essenziale, espressa da Aristotele con la locuzione kath' hauto. Questo specifico rapporto garantirebbe la partecipazione dell'affezione all'essere.

Conformemente al procedimento illustrato, si immagini di dover dimostrare l'esistenza delle determinazioni "pari" e "dispari". Per prima cosa occorre assumere l'esistenza e la definizione dell'oggetto cui ineriscono le due proprietà. Nel caso specifico si tratta della nozione "numero", la quale afferisce all' ambito dell' aritmetica. Poi si presuppone l'espressione definitoria delle nozioni "pari" e "dispari". Terminato il momento prepa-

HA. А 6, 419a13-17).

${ }^{27}$ Il termine ghenos, adoperato nella formulazione della dimostrazione, sembra avere due significati distinti: da una parte identifica l'insieme dei contenuti di una scienza specifica, dall'altra corrisponde al soggetto della dimostrazione. La fluttuazione del significato di ghenos scompare del tutto nello schema fornito in APo. A 10 (in part. 76b12-3), dove Aristotele indica con precisione come elemento della prova gli oggetti che compongono il genere e non il genere in se stesso. Per un approfondimento della questione si rimanda a McKirahan 1992: 50-63.

${ }^{28}$ In APo. A 10, 76b6-11 Aristotele sostiene che la verifica dell'esistenza della proprietà del genere è conseguita attraverso proposizioni comuni (dia) e partendo da proposizioni già dimostrate $(e k)$. La distinzione tra $\mathrm{i}$ due tipi di proposizioni sembrerebbe corrispondere alla riformulazione in ambito teorico del procedimento apodittico proprio delle scienze matematiche dell'epoca e in particolare dell'astronomia, come precisato dallo stesso Aristotele. 
ratorio, si procede dalle proposizioni comuni a manifestare l'appartenenza, in quanto proprietà essenziali, delle affezioni "pari” e "dispari” all'oggetto "numero". Dopo aver mostrato che "ogni numero è pari o dispari", si può concludere che le affezioni pari e dispari esistono ${ }^{29}$.

L'essenzialismo è il tratto caratterizzante della riflessione sulla dimostrazione negli Analitici secondi. La prova scientifica si propone di attestare l'esistenza delle affezioni mediante la loro riconduzione alle sostanze cui ineriscono. Le articolazioni della realtà sono pertanto conoscibili scientificamente solo se inserite in modo appropriato in una struttura logica incentrata sull'essenza di ciascun oggetto. L'attenzione di Aristotele è dunque indirizzata al reperimento di un metodo che permetta l'organizzazione e la sistemazione delle proprietà in riferimento alla specifica sostanza di appartenenza.

La difficoltà principale nell'adozione del modello di APo. A 7 e 10 consiste nel comprendere il ruolo effettivamente svolto dagli assiomi, i quali sono considerati genericamente da Aristotele come principi comuni, poiché trovano applicazione in diversi ambiti scientifici, non appartenendo in modo esclusivo al patrimonio di contenuti di una specifica disciplina. Gli assiomi tuttavia non esibiscono il medesimo livello di generalità e pertanto è possibile operare alcune distinzioni in seno ad essi. In particolare sembra legittimo poter parlare di due ordini di proposizioni identificati dalla parola axioma negli Analitici secondi: le proposizioni appartenenti a più di un genere ${ }^{30} \mathrm{e}$ i principi generalissimi, cioè le leggi logiche del pensiero, che permettono l'acquisizione di conoscenza ${ }^{31}$.

Nel primo gruppo rientra per esempio la proposizione "togliendo uguali da uguali, restano uguali" ${ }^{32}$. Essa accomuna le discipline i cui oggetti hanno attinenza con la nozione di quantità ${ }^{33}$. Al di fuori di questo ambito dell'essere il principio di uguaglianza non possiede alcun valore, poiché non si può riferire ad un oggetto che non sia suscettibile di trattazione

${ }^{29}$ Per un'analisi dettagliata dei passi di APo. A 7 e 10 in cui viene presentato lo schema di inferenza tripartito si rimanda ai seguenti studi: Barnes 1994: 13032, 137-44; Detel 1993: 164-86, 216-35; Mignucci 1975: 136-50, 186-215; Ross 1949: 530-32, 538-41.

${ }^{30}$ Aristot. APo. A 7, 75a41-2; A 10, 76b14-6; A 11, 77a27-8; A 32, 88b27-9.

${ }^{31}$ Aristot. APo. A 2, 72a16-7; A 11, 77a10-2.

${ }^{32}$ Aristot. APo. A 10, 76a41.

${ }^{33}$ Aristot. Metaph. K 4, 1061b18-20. 
quantitativa. Nel secondo gruppo si collocano il principio di contraddizione ${ }^{34}$ e il principio del terzo escluso ${ }^{35}$, i quali hanno una applicazione assoluta, in quanto proposizioni proprie dell'essere in quanto essere, riguardanti cioè ogni genere della realtà. Chiunque conduca una confutazione per assurdo, si serve di questi principi, assumendoli non in forma universale, ma in misura adeguata al genere specifico intorno a cui verte la prova ${ }^{36}$.

Negli Analitici secondi gli assiomi sono pertanto sia parte costitutiva della dimostrazione, in quanto proposizioni ex hon, vale a dire premesse da cui scaturiscono le conclusioni, sia principi generali immediati, che bisogna assumere per apprendere qualcosa e che non figurano direttamente nelle dimostrazioni, se non nel caso delle confutazioni per assurdo. Non è chiaro tuttavia come da leggi logiche o da criteri quantitativi, sebbene presi nelle loro specificazioni disciplinari, sia possibile dimostrare l'esistenza di una affezione, ovvero rendere evidente l'inerenza per sé di una determinazione ad un oggetto. L'assioma non risulta premessa appropriata ad esprimere i contenuti particolari della conclusione a causa del suo elevato livello di generalità. I principi comuni sono quindi incapaci di dare conto dell'appartenenza di un predicato ad un soggetto.

La scelta di collocare gli assiomi al vertice della prova potrebbe essere stata suggerita dalla considerazione dei metodi dimostrativi che, contemporaneamente alla scrittura degli Analitici secondi, stavano prendendo forma negli ambienti matematici dell'Accademia. I frammenti e le testimonianze pervenute attestano un ampio uso, nella verifica degli enunciati, delle proprietà di uguaglianza, ricorrenti nelle esemplificazioni aristoteliche. Nel sistema euclideo inoltre le nozioni comuni, come il principio di uguaglianza, e i postulati, intervengono direttamente ed indirettamente nella dimostrazione di un elevato numero di teoremi. ${ }^{37}$.

Si può supporre che lo schema di inferenza, presentato in APo. A 7 e 10, sia solo un abbozzo, destinato ad essere rielaborato e precisato nelle sue parti. Nel momento in cui esso fu formulato, Aristotele aveva probabilmente già maturato una impostazione essenzialistica della scienza, sebbene a questa acquisizione sul piano teorico non si accompagnasse

${ }^{34}$ Aristot. APo. A 11, 77a10.

${ }^{35}$ Aristot. APo. A 11, 77a22.

${ }^{36}$ Aristot. APo. A 11, 77a22-5; Metaph. Г 3, 1005a25-7; anche Г 3, 1005b15-7.

${ }^{37}$ Sintetiche introduzioni alla matematica greca classica, corredate da ampi riferimenti bibliografici, si trovano in Mueller 1997 e Mueller 2006. 
ancora la definizione sul piano operativo di uno strumento efficace nel fornire espressione concreta alla sua originale concezione epistemologica.

4. Gli elementi dimostrativi definiti nei passi degli Analitici secondi, precedentemente esaminati, appaiono scarsamente integrabili in un unico procedimento. Le difficoltà suscitate dalla lettura dei brani potrebbero però essere risolte ipotizzando nel trattato la presenza di almeno due modelli apodittici, dotati di apparati logici distinti: la deduzione scientifica, delineata principalmente in $A P O$. A 2, 71b9-72a7, e una generale teoria apodittica, abbozzata in APo. A 7, 75a39-b2 e A 10, 76b11-22. Negli Analitici secondi sembrano essersi sedimentati gli stadi della riflessione aristotelica sul procedimento scientifico, la cui costruzione presenta pertanto differenti livelli. I frequenti punti di discontinuità, riscontrabili nell'esposizione della dottrina della scienza, inducono ad ipotizzare la presenza di due strutture dimostrative, le quali non assolverebbero finalità diverse, condizione che ne permetterebbe la coesistenza, ma sarebbero entrambe predisposte ad esibire l'appartenenza per sé di un predicato al rispettivo soggetto.

A sostegno della scarsa compatibilità dei due impianti dimostrativi, illustrati negli Analitici secondi, si può innanzitutto considerare l'eterogeneità, rispetto alla deduzione scientifica, di alcuni elementi apodittici costitutivi del modello di APo. A 7 e 10, quali le proposizioni esistenziali e i principi comuni ${ }^{38}$.

Gli enunciati esistenziali, come per esempio "il numero è", corrispondono ad attribuzioni di essere ad un soggetto e pertanto sono formulati in modo sintatticamente differente rispetto alle proposizioni della deduzione scientifica, le quali sono composte da un predicato che compete per sé al soggetto ed esprimono connessioni del tipo "A appartiene per sé a B". Risulta difficile comprendere come le assunzioni di esistenza del ghenos possano effettivamente trovare posto nella deduzione scientifica.

Una proposizione del tipo "B è", seppure non ammissibile in modo diretto in un sillogismo, potrebbe rappresentare la condizione che garantisce l'assunzione di un genere come soggetto della dimostrazione ${ }^{39}$. Gli enunciati esistenziali avrebbero così una funzione preparatoria alla verifica

${ }^{38}$ L'estraneità degli assiomi e delle proposizioni esistenziali rispetto all'impianto sillogistico è stata messa in luce in Barnes 1981: 40-3.

${ }^{39}$ L'ipotesi è stata avanzata in Mignucci 1975: 187-8. 
dell'esistenza dei nessi predicativi. Aristotele tuttavia, dopo aver presentato le caratteristiche della deduzione scientifica, non si pone il problema dell' esistenza degli oggetti che prendono parte alla dimostrazione e quindi non si preoccupa di trovare una collocazione alle proposizioni esistenziali, che anzi diventano marginali nella sua considerazione. La necessità di saldare le proposizioni esistenziali alla deduzione scientifica non è perciò mai prospettata negli Analitici secondi.

Ugualmente gli assiomi non solo non presentano una struttura sintattica rispondente ai criteri stabiliti da Aristotele per le proposizioni della deduzione scientifica, ma si mostrano riluttanti ad una loro riformulazione conforme al modello sillogistico. Gli assiomi, pur essendo parte fondamentale del procedimento elaborato in APo. A 7 e 10, non possono intervenire in modo diretto nella deduzione scientifica, perché essa non è predisposta ad accogliere proposizioni che esprimono una relazione alternativa alla connessione di natura essenziale tra soggetto e predicato.

Si potrebbe tentare di considerare gli assiomi come un corpo di leggi logiche esterne al sillogismo, le quali, senza prendere esplicitamente parte alla prova, contribuirebbero alla corretta formulazione delle proposizioni scientifiche ${ }^{40}$. Aristotele probabilmente non volle rinunciare a sottolineare il ruolo regolativo svolto da principi come quello di contraddizione o del terzo escluso, nonostante la consapevolezza che nessuna deduzione scientifica può includere espressamente un assioma ${ }^{41}$.

La deduzione scientifica e il modello di prova delineato in APo. A 7 e 10 si avvalgono di impalcature logiche differenti. Nel primo caso si assume il sillogismo come strumento che permette di giungere a conclusioni formalmente valide e contenutisticamente vere. Nel secondo si fa riferimento ad un generico e meno rigoroso schema di inferenza, in cui manca il riferimento alla funzione esplicativa svolta dai principi, ribadita invece a proposito della deduzione scientifica. La concatenazione tra premesse e conclusione presenta pertanto in APo. A 7 e 10 un notevole grado di enigmaticità. Il procedimento appare più accostabile alla formulazione embrionale di un metodo apodittico che ad una compiuta prova dimostrativa.

I due modelli, sebbene strutturalmente divergenti, sono legati da una specifica continuità, la quale sembrerebbe suggerire una evoluzione della

${ }^{40}$ La proposta interpretativa, relativa alla funzione degli assiomi rispetto alla deduzione scientifica, è stata formulata da Ross 1949: 531-2.

${ }^{41}$ Aristot. APo. A 11, 77a10-2. 
teoria aristotelica della dimostrazione. Il metodo di APo. A 7 e 10 e la deduzione scientifica di APo. A 2 si propongono di mostrare l'inerenza per sé di una proprietà ad un genere. Entrambe le dimostrazioni mirano alla riconduzione di ciascuna determinazione alla rispettiva sostanza di appartenenza. Il passaggio da una prima e generica prova di esistenza di un'affezione ad un sillogismo capace di manifestare con evidenza i nessi predicativi proposizionali sembrerebbe porsi in modo plausibile come il progressivo perfezionamento dello strumento metodologico designato per la comprensione scientifica del reale nelle categorie della sostanza e dei suoi accidenti.

Negli Analitici secondi di conseguenza si trovano accostate sia intuizioni e schematiche partizioni sia accurate caratterizzazioni degli elementi apodittici. Le incongruenze, presenti nel trattato, documenterebbero il processo di formazione della dottrina della dimostrazione, il cui momento costitutivo potrebbe essere ravvisato in un preliminare schema assiomatico ed il cui momento conclusivo potrebbe essere individuato in uno preciso modello sillogisticamente strutturato.

Le opere logiche, raccolte nell'Organon, rappresentano la prima trattazione sistematica dedicata al concetto di deduzione e non hanno alcun precedente cui possano venire accostate ${ }^{42}$. Aristotele non si è potuto avvalere di ricerche condotte dai suoi predecessori sulla dimostrazione, ma ha avuto come principale riferimento per l'analisi teorica i procedimenti concretamente applicati dagli studiosi di matematica. La geometria tra la fine del V e il IV secolo si stava indirizzando verso un ideale apodittico altamente formalizzato, rigoroso nella sua struttura e rispondente a specifici rapporti di antecedenza e conseguenza tra le proposizioni. La dimostrazione di un teorema era verosimilmente condotta mediante il ricorso a nozioni comuni, come l'assioma dell'uguaglianza, e a proposizioni proprie, cioè enunciati precedentemente verificati ed assimilati nel corpo dei contenuti della disciplina.

Aristotele poteva attingere direttamente ai metodi di esposizione ed organizzazione del sapere, adottati dai matematici operanti nell'Accademia. È naturale che la riflessione sulla scienza manifesti inizialmente uno stretto legame alla matematica, come attestato negli Analitici secondi sia dalla presenza di definizioni ed esempi tratti dall'aritmetica e dalla geometria, sia dalla distinzione delle proposizioni in comuni a più saperi e proprie di

${ }^{42}$ Aristot. SE. 34, 183b16-184b8. 
una singola disciplina, sia dal ruolo attivo ascritto agli assiomi nella verifica di una proprietà, sia infine dall'andamento deduttivo della dimostrazione, condotta a partire da principi non provati.

L'influenza della geometria sulla dottrina aristotelica della scienza è tuttavia ravvisabile soprattutto nella prima fase di costruzione della teoria, come confermato dalle evidenti analogie tra lo schema abbozzato in APo. A 7 e 10 e le strategie apodittiche di cui si avvalevano gli studiosi del periodo. La deduzione scientifica al contrario mostra un netto affrancamento dai metodi propriamente matematici, soprattutto nell'adozione di un apparato sillogistico, profondamente difforme rispetto ai procedimenti geometrici ${ }^{43}$.

È probabile che nel lavoro di definizione della dimostrazione la logica matematica offrisse ad Aristotele lo spunto per l'elaborazione di un provvisorio modello di inferenza, destinato ad essere successivamente rimpiazzato dall'acquisizione della sillogistica, struttura più adeguata all'impostazione essenzialistica della scienza. Proprio in alcune sezioni degli Analitici secondi Aristotele fornì una prima applicazione del sillogismo, la cui adozione risulta anteriore alla trattazione generale e più dettagliata contenuta negli Analitici primi. Non a caso la teoria aristotelica della scienza non presuppone la fruizione di un ricco e sofisticato apparato sillogistico, ma si avvale di un modello deduttivo estremamente semplice e lineare nei suoi passaggi.

L'ipotesi di una evoluzione della dottrina apodittica aristotelica da un generico schema di inferenza ad un più preciso metodo dimostrativo a carattere sillogistico trova indirettamente riscontro sia nella precedenza, attualmente riconosciuta dalla maggior parte degli studiosi, della teoria della scienza rispetto alla teoria del sillogismo sia nella reciproca influenza di cui le due trattazioni beneficiarono nella fase successiva alla loro preliminare elaborazione $^{44}$. Aristotele, dopo aver abbozzato la teoria apodittica in forma assiomatizzata, ispirandosi in qualche misura ai metodi dimostrativi matematici, avrebbe individuato con la scoperta del sillogismo l'impalcatura logica

${ }^{43}$ L'eterogeneità tra il modello dimostrativo, emergente nella matematica tra la fine del V e il IV secolo, ed il sillogismo è stata ampiamente argomentata in Mueller 1974.

${ }^{44}$ Per un approfondimento della relazione tra gli Analitici primi e gli Analitici secondi e per uno studio dei presupposti sillogistici richiesti alla teoria della scienza negli Analitici secondi si rimanda a Smith 1982a; Smith 1982b; Smith 1989: XIIIXV; Barnes 1981; Barnes 1994: XV-XVIII. 
maggiormente capace di illustrare da un punto di vista metodologico il suo ideale di conoscenza esplicativa e necessaria, cioè propriamente scientifica.

\section{Bibliografia}

Barnes, J. (1981), "Proof and the Syllogism", in E. Berti (ed.), Aristotle on Science. The Posterior Analytics (Proceedings of the Eighth Symposium Aristotelicum). Padova, Antenore: 17-59.

Barnes, J. ed. (1994, 2a ed.), Aristotle. Posterior Analytics. Oxford: Clarendon Press.

Burnyeat, M. (1981), “Aristotle on Understanding Knowledge”, in E. Berti (ed.), Aristotle on Science. The Posterior Analytics (Proceedings of the Eighth Symposium Aristotelicum). Padova, Antenore: 97-139.

Detel, W. Hrsg. (1993), Aristoteles. Analytica posteriora, 2 Halbbände, zweiter Halbband. Berlin: Akademie Verlag.

McKirahan, R. D. (1992), Principles and Proofs. Aristotle's Theory of Demonstrative Science. Princeton: Princeton University Press.

Mendell, H. (1988), "Making Sense of Aristotelian Demonstration", OSAPh 16: 161-225.

Mignucci, M. (1975), L'argomentazione dimostrativa in Aristotele. Commento agli Analitici secondi. Padova: Antenore, 1975.

Mignucci, M. (2007), Aristotele. Analitici secondi. Organon IV. Roma Bari: Laterza.

Mueller, I. (1974), "Greek Mathematics and Greek Logic", in J. Corcoran (ed.), Ancient Logic and its Modern Interpretation, Dordrecht, Reidel: 35-70.

MuelLeR, I. (1997), "Greek arithmetic, geometry and harmonics: Thales to Plato", in C. C. W. Taylor (ed.), Routledge History of Philosophy, vol. 
I, From the Beginning to Plato, London and New York, Routledge: 271-322.

Mueller, I. (2006), "Greek Mathematics (Arithmetic, Geometry, Proportion Theory) to the Time of Euclid", in M. L. Gill and P. Pellegrin (eds.), A Companion to Ancient Philosophy. Oxford - Boston, Blackwell: 686-718.

Ross, W. D. ed. (1949), Aristotle's Prior and Posterior Analytics. Oxford: Clarendon Press.

Smith, R. (1982a), "The Relationship of Aristotle's Two Analytics", $C Q$ 32: $327-35$.

Smith, R. (1982b), "The Syllogism in the Posterior Analytics I", AGPh 64: 113-35.

Sмiтh, R. ed. (1989), Aristotle. Prior Analytics, Indianapolis: Hackett. 